\title{
Les querelles de mots dans le discours politique : modèle d'analyse et étude de cas à partir d'une polémique sur le mot « rigueur »
}

Verbal Disputes in Political Discourse: A Model of Analysis and a Case Study of a Controversy over the Word "Rigueur"

\section{Raphaël Micheli}

\section{(2) OpenEdition Journals}

\section{Édition électronique}

URL : http://journals.openedition.org/aad/1446

DOI : $10.4000 /$ aad. 1446

ISSN : 1565-8961

\section{Éditeur}

Université de Tel-Aviv

\section{Référence électronique}

Raphaël Micheli, « Les querelles de mots dans le discours politique : modèle d'analyse et étude de cas à partir d'une polémique sur le mot « rigueur » », Argumentation et Analyse du Discours [En ligne], 10 | 2013, mis en ligne le 10 avril 2013, consulté le 23 septembre 2019. URL : http:// journals.openedition.org/aad/1446; DOI : 10.4000/aad.1446

Ce document a été généré automatiquement le 23 septembre 2019.

\section{cc) $(1) \odot$}

Argumentation \& analyse du discours est mis à disposition selon les termes de la licence Creative Commons Attribution - Pas d'Utilisation Commerciale - Pas de Modification 4.0 International. 


\section{Les querelles de mots dans le discours politique : modèle} d'analyse et étude de cas à partir d'une polémique sur le mot « rigueur »

Verbal Disputes in Political Discourse: A Model of Analysis and a Case Study of a Controversy over the Word "Rigueur"

Raphaël Micheli

\section{Les querelles de mots : un objet à la croisée des études sur le métalangage, l'argumentation et la communication politique}

L'objectif de cet article est de contribuer à la description d'un type particulier d'affrontement qui met régulièrement aux prises les acteurs politiques dans l'espace médiatique contemporain : la querelle de mots. Ce type d'affrontement émerge lorsque l'usage qu'un acteur politique fait d'un mot est jugé remarquable, tant par les journalistes que par d'autres acteurs politiques. La visibilité dont bénéficie soudainement le mot peut tenir à des raisons très diverses. L'acteur politique en question peut, par exemple, utiliser de manière ostensible un mot dont son parti répugne traditionnellement à faire usage ${ }^{1}$. Il peut aussi, à l'inverse, récuser un mot que ses adversaires, voire ses alliés, l'incitent à employer pour désigner telle ou telle mesure politique. Quel que soit le cas de figure envisagé, un mot particulier acquiert une forte saillance politico-médiatique, et cela en un laps de temps généralement très court. Par un travail conjoint des acteurs politiques et des journalistes, le mot vient occuper le devant de la scène, dans la mesure où il est érigé en objet du désaccord : il 
devient, pour une durée médiatique limitée, ce à propos de quoi il y a lieu de s'affronter. La question à propos de laquelle les journalistes invitent les acteurs politiques à croiser le fer tourne en effet autour de la pertinence de l'usage d'un mot dans un contexte donné : « Tel acteur politique a utilisé le mot X ; selon vous, fautil ou ne faut-il pas l'utiliser? " Les journalistes se livrent ainsi à un travail de cadrage médiatique : ils poussent les acteurs politiques non seulement à commenter la façon dont leurs pairs utilisent le mot, mais aussi à prendre personnellement position quant à son usage. Engagés pour leur part dans un travail de positionnement, les acteurs politiques s'efforcent de justifier par des raisons la position prise à l'égard du mot : il s'agit alors pour eux d'argumenter pour ou contre l'usage du mot.

Pour le chercheur, ces querelles de mots constituent des objets dont l'étude s'avère extrêmement riche sur le plan scientifique : il y a là, en effet, l'occasion d'articuler d'une façon originale les sciences du langage, les théories de l'argumentation et les sciences de la communication et des médias. Je pars ici de l'hypothèse que les querelles de mots offrent au chercheur un terrain privilégié pour décrire comment l'activité métalinguistique des locuteurs trouve à s'exercer dans le cadre spécifique d'argumentations relevant du discours politique médiatisé. Le défi méthodologique est de parvenir à intégrer en une démarche aussi cohérente que possible trois questions qui sont le plus souvent traitées séparément: celle du pouvoir de réflexivité des langues (ici le français), celle du fonctionnement des discours argumentés et, enfin, celle de la mise en scène médiatique de la parole des acteurs politiques. Par rapport à l'état de la recherche ${ }^{2}$ dans les trois domaines signalés, l'étude des querelles de mots me semble présenter un triple intérêt :

3 (1) Elle se rattache d'abord au domaine bien constitué de l'étude des formes du métalangage ordinaire et de leurs usages discursifs. Dans ce domaine, il s'agit, à un niveau théorique, d'établir une typologie raisonnée des formes-nombreuses et variées - au moyen desquelles il est possible, pour les locuteurs, de constituer le langage en objet et d'en dire quelque chose ${ }^{3}$. Il s'agit également, à un niveau davantage empirique, de décrire comment l'activité métalinguistique des locuteurs se manifeste en fonction des normes régissant un genre de discours ou un contexte de communication particulier ${ }^{4}$. L'intérêt que présente ici l'étude des querelles de mots tient à ce qu'elle se propose d'explorer les rapports entre, d'une part, l'activité métalinguistique que les locuteurs déploient à propos d'un mot - et qui se traduit par le recours à diverses formes du métalangage ordinaire - et, d'autre part, l'argumentation que les locuteurs construisent à propos de ce mot : c'est là un croisement qui est encore relativement peu exploré par les linguistes s'intéressant à la réflexivité langagière.

(2) Dans le champ des théories de l'argumentation, de nombreuses approches tendent à voir les mots comme des instruments imparfaits dont les locuteurs doivent s'efforcer de régler l'usage afin de justifier leur point de vue d'une manière aussi claire et " raisonnable » que possible. L'ambiguïté des mots et les multiples « connotations » qu'ils charrient sont alors perçues comme des obstacles qui, bien qu'importants, peuvent être surmontés si les locuteurs engagés dans l'échange argumentatif prennent la peine de s'entendre sur le sens qu'ils octroient aux termes utilisés ${ }^{5}$. Avec l'étude des querelles de mots, on s'intéresse à des cas où les mots ne peuvent absolument pas être considérés comme de purs instruments au service de l'argumentation, dans la mesure où ils en deviennent les enjeux mêmes. Comment se déroule typiquement un échange argumentatif lorsqu'il se concentre sur un mot? Par quels processus argumentatifs la 
justification d'une position favorable ou défavorable à l'usage du mot litigieux se caractérise-t-elle au juste ? C'est là un aspect encore mal connu du fonctionnement discursif de l'argumentation ${ }^{6}$.

5 (3) Enfin, l'étude des querelles de mots touche à la question de la médiatisation de la parole politiqueet concerne donc aussi les sciences de la communication et des médias. Elle peut, à ce titre, mettre en évidence certains aspects importants du travail de cadragequ'opèrent les journalistes lorsqu'ils mettent en scène le discours des acteurs politiques, notamment la tendance à en extraire des séquences verbales brèves à des fins de spectacularisation ${ }^{7}$. Elle devrait aussi montrer que le positionnement des acteurs politiques dépend en partie de la conquête d'un lexique qui leur est propre: l'affrontement politique dans les médias voit alors inévitablement les mots devenir des objets de lutte.

6 Au niveau de sa démarche méthodologique, le présent article se veut tout à la fois théorique et empirique. Il entend en effet esquisser un modèle d'analyse des querelles de mots et, sur la base d'un exemple concret, en évaluer la pertinence pour l'analyse des données discursives. La première partie de l'article sera consacrée à une présentation succincte de ce modèle (2.). Dans la seconde partie (3.), je procéderai à l'analyse d'un corpus constitué de discours relevant d'une polémique qui, entre mai et juillet 2010, a opposé plusieurs acteurs politiques français autour de l'usage du mot « rigueur ». Le 6 mai, le Premier Ministre François Fillon annonce une série de mesures (comme le gel des dépenses de l'Etat durant trois ans) et d'objectifs (comme le fait de ramener le déficit public sous la barre des $3 \%$ d'ici à 2013). Si la pertinence du mot «rigueur» pour désigner une telle politique est généralement reconnue par l'opposition socialiste, les principaux représentants de l'UMP (membres ou non du gouvernement) se montrent profondément divisés : certains, à l'instar de Jean-François Copé ou Alain Juppé, prônent l'usage du mot litigieux, tandis que beaucoup d'autres - le Premier Ministre en tête, ainsi que plusieurs membres de son gouvernement - le récusent avec force. La polémique se complexifie et s'intensifie entre le 14 et le 19 juillet: Nicolas Sarkozy rejette le mot durant son intervention le jour de la fête nationale, mais François Fillon l'utilise finalement le 16 lors d'un déplacement, et, interrogé le 19 par des journalistes, affirme ne pas «regretter» de l'avoir fait. Au niveau générique, ce corpus se compose majoritairement d'interviews (radio- et télédiffusées) et de conférences de presse lors desquelles les acteurs politiques sont interrogés par des journalistes. On s'intéresse donc prioritairement aux interventions orales des acteurs politiques dans des contextes médiatiques de type dialogal où le discours est polygéré. Le corpus inclut aussi, plus marginalement, les interventions écrites des acteurs politiques dans des contextes de type monologal: on pense particulièrement au genre du blog ou de l'article d'opinion dans la presse écrite. Je précise enfin que, pour des questions de faisabilité, le corpus traité dans le cadre de cet article reflète une séquence politico-médiatique limitée dans le temps : il est clair qu'il pourrait être enrichi sur une plus longue durée, au vu de l'embarras qu'a semblé susciter, tout juste deux ans plus tard, le mot "rigueur" chez les responsables socialistes arrivés au pouvoir suite à l'élection présidentielle de mai 2012. 


\section{Esquisse d'un modèle d'analyse}

7 Je commencerai par présenter brièvement un modèle permettant l'analyse des querelles de mots qui opposent les acteurs politiques dans les médias contemporains. L'enjeu est, plus précisément, de pouvoir rendre compte du fonctionnement de l'argumentation lorsque le désaccord se cristallise autour d'un mot donné et que les locuteurs construisent une position favorable ou défavorable à l'usage de ce mot en la justifiant au moyen d'arguments. Ce modèle d'analyse est volontairement " économique ", dans la mesure où il ne multiplie pas les entrées et repose sur trois axes. J'espère montrer que l'économie du modèle est l'une des conditions de sa rentabilité sur le plan empirique de la description des corpus. L'objectif, en effet, est de se concentrer sur un nombre limité de processus discursifs qui sont à ce point récurrents dans les querelles de mots qu'ils semblent y exercer une fonction véritablement structurante : on les verra à l'œuvredans la polémique autour du mot " rigueur ", mais il faudrait à terme en éprouver la pertinence sur d'autres querelles de mots. J'offre, dans cette section, une présentation très succincte des trois axes, qui seront repris, détaillés et exemplifiés lors de la description du corpus (infra,3.).

8 Le premier axe de l'analyse s'intéresse au travail de cadrage médiatique qu'opèrent les journalistes afin d'inciter les acteurs politiques à prendre position par rapport à l'usage du mot litigieux. Il s'agit ici d'examiner de quelle manière les journalistes participent à l'émergence d'une querelle de mots et contribuent à ce que ladite querelle soit «alimentée » durant un certain laps de temps. Le genre de l'interview se prête tout particulièrement à l'observation de ce travail de cadrage : le mot devient ce à propos de quoi il y a lieu de prendre position,sous l'influence des commentaires introductifs auxquels se livrent les journalistes et, surtout, des stratégies de questionnement qu'ils déploient lorsqu'ils interrogent les acteurs politiques (infra,3.1.).

9 Lors d'une querelle de mots, les acteurs politiques sont amenés à prendre position quant à l'usage du mot qui fait problème. Le deuxième axe de l'analyse concerne ainsi les moments où les acteurs politiques explicitent, sur un mode réflexif, leur attitude à l'égard du mot en question. L'enjeu est ici de décrire dans le détail comment se formulent de telles prises de position, à la fois auto- et allo-centrées, et qui mêlent de façon complexe descriptionet prescription: en effet, un acteur politique donné dit ce qu'il fait lui-même du mot, ce que d'autres que lui font du mot et, ultimement, ce qu'il faut faire du mot (infra, 3.2.).

10 Avec le troisième axe de l'analyse, on touche à la question de l'argumentation: les acteurs politiques tentent en effet de justifier leur prise de position à l'égard de l'usage d'un mot en formulant les raisons pour lesquelles ce mot doit (ou ne doit pas) être utilisé. Comme je tâcherai de le montrer, la construction d'une argumentation pour ou contre l'usage d'un mot intègre fondamentalement trois composantes majeures : (1) la question du sens de ce mot; (2) la question de son applicabilité référentielle ; (3) la question de son dialogisme. (1) Premièrement, lorsqu'ils tentent d'étayer leur position à l'égard d'un mot, les locuteurs disent ce que le mot signifie pour eux. Ils s'engagent dans des opérations de spécification du sens qui peuvent prendre des formes très variées. Dans une telle optique, on s'arrêtera notamment sur le problème de la définitionet l'on examinera les différents types d'énoncés définitoires auxquels les locuteurs ont recours. (2) Deuxièmement, la question du sensdu mot est intimement liée à celle de son applicabilité référentielle : les locuteurs vont alors dire si le mot est 
apte ou non à désigner un certain objet du monde. (3) Troisièmement, la question du dialogisme se pose inéluctablement: comme le dit Bakhtine, dans une formule restée célèbre, « chaque mot renvoie à un contexte ou à plusieurs, dans lesquels il a vécu son existence socialement sous-tendue » (1978: 114). Il faudra, à ce titre, voir comment les locuteurs tentent d'exploiter argumentativement la charge dialogique du mot pour ou contre lequel ils prennent position. Mon hypothèse est que l'étude des querelles de mots permet de dégager des types d'enchainements argumentatifs relativement stables et récurrents, auxquels les locuteurs ont recours afin de justifier l'usage ou le nonusage du mot litigieux. Je décrirai, sur la base de quelques exemples, deux de ces enchaînements prototypiques, en montrant comment ils intègrent les trois composantes du sens, de la référence et du dialogisme (infra,3.3.).

\section{Approche du déroulement argumentatif des querelles de mots à travers l'exemple de la polémique sur le mot « rigueur »}

\subsection{Le cadrage médiatique de la " querelle de mots " : commentaires et questions des journalistes}

11 De quelle manière les journalistes constituent-ils un mot en objet de désaccord, à propos duquel les acteurs politiques sont invités (voire acculés) à prendre position? On tentera de saisir ce travail de cadrage à travers les commentaires introductifs auxquels se livrent les journalistes avant l'interview d'un acteur politique et à travers le type de questions qu'ils lui adressent.

Les commentaires introductifs consistent le plus souvent à présenter l'attitude des acteurs politiques à l'égard du mot sous l'angle de l'embarras :

(1) rigueur ou non/ rigueur le mot fait peur à ceux qui nous gouvernent (Début de

l'interview de Michel Sapin, 7 mai 2010) ${ }^{8}$

13 - ou de mettre au jour une discrépance entre les mots (que l'on observe de façon tangible dans les discours tenus publiquement par les acteurs politiques) et l'intention (que l'on peut plausiblement attribuer à ces acteurs au vu de la politique qu'ils mènent) :

(2) JOURN1 : le buzz politique du jour c'est Nicolas Sarkozy qui veut faire la rigueur mais en silence

JOURN2 : y penser toujours n'en parler jamais... (Début de l'interview de JeanFrançois Copé, 10 mai 2010) ${ }^{9}$

Lorsqu'une question est directement adressée au responsable politique, elle peut :

- (1) porter sur l'attitude personnelle du locuteur à l'égard du mot :

(3) ce mot a l'air de faire peur à tout le monde est-ce qu'il vous fait peur ce mot

rigueur/ (Interview de François Baroin, 14 mai 2010) ${ }^{10}$

(4) et le mot rigueur ne vous fait pas peur/ (Interview de Nicolas Sarkozy, 14 juillet 2010) ${ }^{11}$

- (2) porter sur l'attitude personnelle du locuteur à l'égard du mot en tant qu'elle s'oppose à l'attitude d'autres acteurs politiques. Le corpus fournit deux belles illustrations de cette stratégie, au gré des changements d'attitude du Premier Ministre François Fillon à l'égard du mot « rigueur ». En (5), Fillon est interrogé sur le fait qu'il refuse le mot, puis en (6) sur le fait qu'il l'a finalement adopté : 
(5) on a compris qu'il fallait pas parler de plan de rigueur à [...] propos [des mesures annoncées] mais qu'est-ce que vous répondez à ceux qui dans votre camp utilisent ce terme euh comme Jean-François Copé comme Alain Juppé semblant vouloir dire que le gouvernement se cache un peu derrière son petit doigt en n'utilisant pas ce mot de rigueur (conférence de presse de François Fillon, 11 mai 2010)

(6) au Japon vous avez parlé de rigueur vous semblez avoir euh cassé un tabou dans l'exécutif puisque lundi encore Nicolas Sarkozy avait récusé ce terme lors de son intervention télévisée est-ce que vous maintenez ce terme euh rigueur/ (conférence de presse de François Fillon, 19 juillet 2010)

Ici, les questions ont une charge polémique évidente, dans la mesure où elles pointent des dissensions entre le locuteur et d'autres représentants du même parti politique relativement à l'usage du mot. Dans le premier cas, la question pousse Fillon à devoir justifier le non-usage d'un mot que des figures proéminentes de son parti utilisent ouvertement. Dans le second cas - et le potentiel polémique est alors encore plus fort -, les dissensions concernent les deux principaux représentants du pouvoir exécutif (le Premier Ministre et le Président de la République). La question cherche à contraindre Fillon soit à confirmer son usage du mot (au risque de contredire publiquement le chef de l'Etat), soit à l'infirmer (au risque de sembler se dédire). Une variante de ce genre de question consiste à prendre à témoin un représentant d'un parti politique (ici encore l'UMP) et de lui demander d'expliquer les dissensions qui rongent son propre campainsi cette question adressée au ministre de la Défense Gérard Longuet, après le revirement de Fillon :

(7) pourquoi est-ce que le président n'ose pas utiliser le mot rigueur pourquoi pas dire la vérité aux Français comme vient de le faire François Fillon le premier ministre (Interview de Gérard Longuet, 16 juillet 2010) ${ }^{12}$

Une telle question cherche à placer l'acteur politique dans une situation de dilemme : quelle que soit sa réponse, il peut difficilement éviter de donner tort à l'une des deux têtes du pouvoir exécutif (la réponse de Longuet est analysée en 3.3.2.).

\subsection{La prise de position des acteurs politiques par rapport à l'usage ou au non-usage du mot}

17 Il s'agit maintenant de voir comment les acteurs politiques prennent position par rapport à l'usage du mot. Dans ce genre de cas, les locuteurs adoptent une attitude réflexive qui se marque de façon tout à fait explicite par l'autonymie : le mot devient «l'objet même du dire » (selon l'expression d'Authier-Revuz 2003: 72) - ce que permet notamment, on le verra, l'emploi de «présentateurs métalinguistiques » : « le mot (de) $\mathrm{X}$ », « ce terme », etc. Comment se formulent typiquement de telles prises de position? On va voir que les possibilités sont ici assez nombreuses, ce qui tient à deux variables :

- Le locuteur peut formuler une prise de position à l'égard de l'usage du mot en prenant pour objet son propre usage ou en commentant l'usage qu'un autre locuteur fait du mot (les deux possibilités n'étant bien sûr pas incompatibles et pouvant être exploitées en parallèle);

- Pour qu'il y ait véritablement prise de position, l'usage du mot ne doit pas simplement être constaté : il doit aussi être évalué, voire recommandé. On peut distinguer les différentes prises de position selon cette seconde variable : à quel degré affichent-elles (ou, au contraire, masquent-elles) les dimensions axiologique et prescriptive dans leur discours sur l'usage du mot? 
conjonction de l'autonymie et d'un verbe comme « utiliser » ou « employer » :

(8) ben moi le mot que j'utilise c'est le mot de rigueur (Interview de Jean-François

Copé, 10 mai 2010)

(9) je n'emploierai absolument pas le mot de rigueur (Point presse de Frédéric Lefebvre, 10 mai 2010) $)^{13}$

De manière plus oblique, le locuteur peut souligner son propre acte d'assertion (« moi je dis que... »), dont le contenu inclut le mot litigieux, et l'opposer à d'autres actes d'assertion possibles sur fond d'un conflit de nomination («appelez ça comme vous voulez ») :

(10) Et la vérité, aujourd'hui, c'est que le niveau d'endettement de notre pays est tel qu'il est urgent de réduire les dépenses publiques. Appelez ça comme vous voulez, moi je dis qu'il faut gérer l'argent public avec une très grande rigueur (Chronique de Jean-François Copé, 18 mai 2010) ${ }^{14}$

On ajoutera que cette description que le locuteur donne de son propre usage peut glisser vers la promesse, en ce sens que le locuteur - produisant un acte de langage de type commissif - s'engage à effectuer une action dans l'avenir, à savoir celle d'utiliser le mot. C'est le cas de François Fillon, après qu'il a utilisé publiquement (le 16 juillet) le mot qu'il récusait depuis plusieurs semaines :

(11) je répéterai [ce mot] à chaque fois que j'en aurai l'occasion (Conférence de presse de François Fillon, Nouméa, 19 juillet 2010)

Bien sûr, le fait d'isoler de tels énoncés de leur cotexte est un peu artificiel. En effet, dans la mesure où elle se voit toujours justifiée par des raisons (dimension argumentative qui sera traitée au point 3.), la description que le locuteur donne de son propre usage se charge presque immanquablement - fût-ce implicitement - d'une dimension prescriptive. Encore en-deçà d'un jugement de valeur ou d'une prescription, l'expression d'une attitude affective du locuteur vis-à-vis de l'usage du mot est fréquente - ainsi Nicolas Sarkozy qui verbalise son ordre de préférence entre les mots « rigueur » et « rigoureux »:

(12) alors le mot rigueur est connoté en France ce qui ne me fait pas peur c'est le mot rigoureux (Interview de Nicolas Sarkozy, 14 juillet 2010) ${ }^{15}$

- ou François Fillon, toujours dans le contexte de l'explication a posteriori de son usage d'un mot qu'il a longtemps refusé :

(13) je n'ai pas peur d'utiliser ce mot je ne le regrette pas (Conférence de presse de François Fillon, 19 juillet 2010)

Deuxième cas de figure, lorsqu'un jugement est explicitement formulé, il porte en général sur l'usage qu'un autre locuteurfait du mot - ainsi ce jugement positif porté par le ministre de la Défense Gérard Longuet sur l'usage du mot par François Fillon :

(14) eh bien je trouve que Fillon a raison d'utiliser un mot qui rassure les marchés (Interview de Gérard Longuet, 16 juillet 2010)

Le jugement peut, de manière plus indirecte, résulter d'une explication de l'usage qu'autrui fait du mot - ainsi cet extrait d'une interview du socialiste Michel Sapin, où le jugement de valeur négatif porté sur l'usage découle du fait qu'il repose, selon le locuteur, sur de mauvaises raisons (en l'occurrence : régler l'usage ou le non-usage du mot en fonction des consignes du chef plutôt qu'en fonction de son degré d'ajustement au réel) :

(15) si la la droite [...] refuse d'utiliser le mot rigueur c'est parce que Nicolas Sarkozy a dit avec moi y aura jamais la rigueur donc ils sont prisonniers en quelque 
sorte de la déclaration du président de la république (Interview de Michel Sapin, 7 mai 2010)

Enfin, troisième cas de figure, la prise de position quant à l'usage peut se faire ouvertement prescriptive : le locuteur enjoint alors à l'allocutaire d'adopter un certain comportement, à savoir d'utiliser ou de ne pas utiliser le mot - ainsi Fillon face aux journalistes, lors de sa phase initiale de refus du mot:

(16) de la même façon que pendant deux ans vous avez c'est un vous collectif évoqué une récession qui n'existait pas euh ne refaites pas la même erreur euh en parlant d'un plan de rigueur qui n'existe pas(Fillon, 7 mai 2010)

- ou Jean-François Copé, défendant la position inverse :

(17) Ne dramatisons pas à l'excès l'emploi du mot rigueur. (Chronique de JeanFrançois Copé, 18 mai 2010)

\subsection{Comment justifier une position prise par rapport à l'usage du mot ? Deux enchaînements argumentatifs prototypiques}

On vient de décrire quelques-unes des modalités selon lesquelles s'opère la prise de position des acteurs politiques quant à l'usage du mot. Il faut maintenant faire un pas de plus et voir comment les locuteurs s'efforcent de justifier leurs prises de position en les étayant par des arguments. Je tenterai, dans ce qui suit, de dégager deux formes d'argumentation qui se trouvent mobilisées de façon récurrente par les acteurs politiques engagés dans la polémique sur le mot "rigueur", et que l'on serait certainement susceptible de retrouver dans d'autres querelles de mots.

\subsubsection{La justification de l'usage ou du non-usage du mot par l'argument de l'applicabilité référentielle}

Le premier type d'enchaînement argumentatif que l'on peut dégager consiste, pour le locuteur, à justifier l'usage (ou le non-usage) du mot en tirant argument du fait que le mot, selon une définition qui en est donnée, s'applique (ou ne s'applique pas) au référent dont il est question. On peut, pour cet enchaînement argumentatif, parler d'une justification de l'usage ou du non-usage du mot par l'argument de l'applicabilité référentielle. Afin d'en expliciter la logique, je m'appuierai sur le schéma suivant, dont je proposerai ensuite un commentaire détaillé :

Etape 1 : Spécification du sens du mot

Prémisse 1 : Le mot $X$ désigne $Y$

(énoncé définitoire) Le mot $X$ signifie $Y$

Un/le X (c')est un/le Y

Etape 2 : Comparaison du sens spécifié avec les caractéristiques du référent

Prémisse $2:$ Or le référent $R$ satisfait / ne satisfait pas aux exigences de la définition du mot X

Etape 3 : Affirmation de l'(in)applicabilité référentielle du mot

Concl. intermédiaire: $\mathrm{R}$ peut/ne peut pas être inscrit dans la classe de référents

dénotée par le mot $X$

(nouvelle prémisse) $\mathrm{R}$ est/ n'est pas un $\mathrm{X}$

[Loi de passage ]:Si un mot est / n'est pas applicable au référent dont il est question,

il doit/ne doit pas être utilisé

Etape 4 : Prise de position quant à l'usage du mot

Conclusion : Le mot X doit / ne doit pas être utilisé 
ce schéma le laisse entrevoir, l'enchaînement argumentatif dont il est question implique quatre étapes distinctes ${ }^{16}$. La première consiste en une spécification du sens : le locuteur produit alors un énoncé définitoire ${ }^{17}$ qui assigne un sens au mot litigieux. La deuxième étape consiste à comparer le sens du mot, tel qu'il vient d'être spécifié par l'énoncé définitoire, avec les traits les plus saillants du référent qu'il s'agit de nommer. Comme le résume bien Plantin (1996: 52 ${ }^{18}$ ), il s'agit ainsi de «confronter les caractéristiques propres » du référent «aux exigences de la définition ». Sur le plan argumentatif, on peut voir ici une deuxième prémisse qui va permettre de relier la première prémisse (spécification du sens) à une conclusion intermédiaire. Ce qui est en jeu lors de la troisième étape, c'est - sur la base des prémisses - de conclure à l'inscription ou à la non-inscription du référent dans la classe des référents dénotée par le mot litigieux et, ainsi, de statuer sur l'applicabilité référentielle du mot. Le degré de congruence entre le sens spécifié et les traits les plus saillants du référent détermine la possibilité ou l'impossibilité de désigner ce référent par le mot en question. Lors de la quatrième et dernière étape de l'argumentation, la conclusion intermédiaire est - si l'on veut - reconvertie en prémisse pour étayer la conclusion finale. Celle-ci formule typiquement une prise de position quant à l'usage du mot, qui peut être favorable ou défavorable. Le passage de la conclusion intermédiaire, reconvertie en prémisse, à la conclusion finale repose sur une loi de passage implicite qui corrèle le degré d'application référentielle d'un mot dans un contexte donné à la pertinence de son usage : [Si un mot est/n'est pas applicable référentiellement, alors il faut/il ne faut pas l'utiliser].

Considérons à présent un exemple (18) de cet enchaînement argumentatif. Il s'agit d'une conférence de presse donnée par François Fillon le 7 mai 2010. A une journaliste qui lui demande si, au vu des «mesures drastiques pour lutter contre le déficit » qu'il vient d'annoncer, le mot "rigueur» est toujours «tabou en France», le Premier Ministre répond ceci :

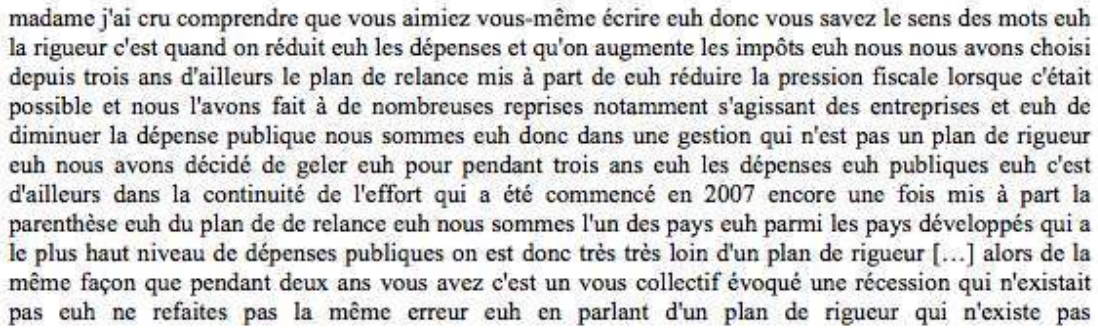

Fillon commence par formuler un énoncé définitoire qui spécifie le sens qu'il convient selon lui d'octroyer au mot: "la rigueur c'est quand on réduit euh les dépenses et qu'on augmente les impôts» (1.1-2). Il s'agit en l'occurrence d'un énoncé définitoire copulatif, dont la particularité syntaxique est qu'il met en rapport, par le biais du verbe «être », un syntagme nominal («la rigueur») et deux propositions coordonnées (" quand on réduit euh les dépenses et qu'on augmente les impôts »). L'étape suivante repose sur la comparaison entre le sens du mot tel qu'il vient d'être spécifié et les caractéristiques du référent qu'il s'agit de nommer, à savoir la politique gouvernementale. Fillon s'efforce de faire apparaître ici deux discrépances. D'une part, le premier trait dégagé dans la spécification du sens ("on augmente les impôts») s'oppose au choix de "réduire la pression fiscale » (1.3), dont Fillon fait l'une des orientations majeures de l'action de son gouvernement. D'autre part, le second trait du 
sens du mot («on réduit les dépenses») s'oppose au fait que «nous sommes l'un des pays euh parmi les pays développés qui a le plus haut niveau de dépenses publiques " (1.7-8) - on note toutefois que cette seconde opposition est moins tranchée que la première, dans la mesure où Fillon affirme également à plusieurs reprises le choix de « diminuer » (1.4-5) ou de "geler» (1.6) les dépenses publiques. Quoi qu'il en soit, cette double opposition permet au Premier Ministre de conclure par deux fois à l'impossibilité d'inscrire le référent dans la classe de référents dénotée par le mot - voir ici l'emploi du connecteur « donc » (« nous sommes euh donc dans une gestion qui n'est pas un plan de rigueur », 1.5, et " on est donc très très loin d'un plan de rigueur ", 1.9). Enfin, la conclusion finale tire parti de l'inapplicabilité référentielle du mot pour formuler une prise de position négative du locuteur par rapport à son usage. En l'occurrence, cette prise de position prend un tour injonctif, le Premier Pinistre usant ici d'un impératif adressé aux journalistes : «alors de la même façon que pendant deux ans vous avez c'est un vous collectif évoqué une récession qui n'existait pas euh ne refaites pas la même erreur euh en parlant d'un plan de rigueur qui n'existe pas » $(1.9-11)$.

\subsubsection{Justifier l'usage ou le non-usage du mot par l'argument de la charge dialogique du mot et de ses effets sur l'allocutaire}

On a vu que pour justifier l'usage ou le non-usage d'un mot, les locuteurs recourent à un enchaînement argumentatif prototypique qui exploite tout à la fois la question du sens de ce mot et celle de son applicabilité référentielle. Il s'agit à présent de considérer un second type d'enchaînement argumentatif qui, cette fois, tire parti du dialogisme du mot litigieux. J'entends ici par "dialogisme ${ }^{19}$ le fait que l'usage d'un mot dans un discours donné évoque immanquablement d'autres discours au sein desquels il a été utilisé. Comme le résument Bres et Nowakowska, «les mots du lexique ne sont jamais “vierges" [...]: ils sont, de façon plus ou moins saillante, gros des énoncés ou des discours qui les ont actualisés » (2006: 33). Il y a là, selon l'expression de Siblot, un dialogisme de la nomination: en nommant un objet, le locuteur se positionne «à l'égard d'autres locuteurs, lesquels nomment autrement ou pareillement l'objet" (Détrie et al. 2001: 207). Comment un tel dialogisme peut-il être mobilisé par les locuteurs lorsqu'il s'agit d'argumenter pour ou contre un mot? Tentons de modéliser les étapes de cet enchaînement argumentatif. Le point de départ de l'argumentation consiste, pour le locuteur, à cibler un certain type d'allocutaire et à affirmer que l'usage du mot produit sur lui des effets (positifs ou négatifs), et cela en vertu du fait que l'usage du mot est dialogiquement associé par cet allocutaire à tel ou tel contexte antérieur d'emploi. L'étape suivante - généralement implicite, mais sans laquelle l'argumentation n'aurait pas de sens - concerne l'importance accordée au point de vue de cet allocutaire. Comme il s'agit, in fine, de tirer une conclusion pratique relative à l'usage du mot sur la base des effets que ce mot produit sur un type d'allocutaire, il est bien entendu nécessaire que le point de vue de celui-ci soit jugé important. Enfin, l'étape conclusive voit le locuteur prendre position relativement à l'usage du mot. Selon le caractère positif ou négatif des effets escomptés sur l'allocutaire, le locuteur affirme que le mot doit ou, au contraire, ne doit pas être utilisé. Le passage des prémisses à la conclusion repose sur une loi de passage typique de l'argumentation par les conséquences (Walton 2006: 104-106) et qui corrèle la nature des conséquences d'une action avec la légitimité de son accomplissement: [Plus une action exerce des 
conséquences positives/négatives, plus/moins il est légitime de l'accomplir]. Pris dans sa globalité, l'enchaînement argumentatif ${ }^{20}$ peut être schématisé ainsi :

Prémisse 1 : L'usage du mot $\mathrm{X}$ exerce, en raison de son dialogisme, des effets positifs

/ négatifs sur un certain type d'allocutaire

Prémisse 2: Il est important de prendre en compte les effets exercés sur ce type d'allocutaire

[Loi de passage]: [Plus une action exerce des conséquences positives/négatives, plus/moins il est légitime de l'accomplir]

Conclusion : le mot X doit / ne doit pas être utilisé (16 juillet 2010), suite au rejet du mot « rigueur » par Nicolas Sarkozy le 14 juillet et à son usage par le Premier Ministre quelques jours plus tard :

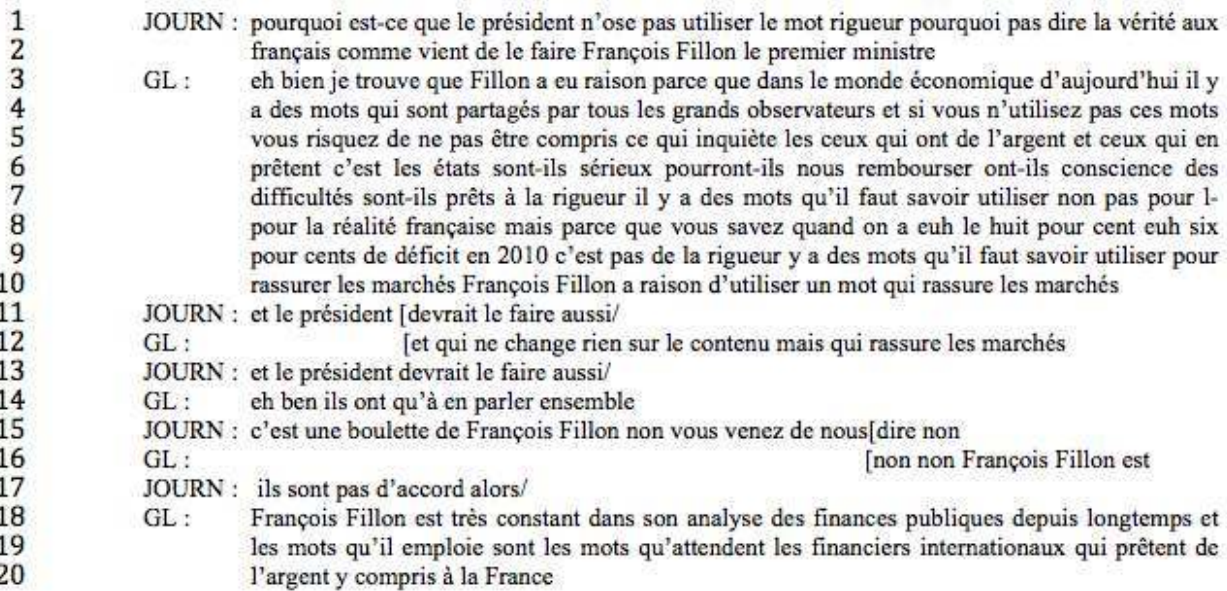

Dans cet extrait, la conclusion est livrée d'entrée, dans la mesure où le locuteur formule une prise de position par rapport à l'usage du mot. Cette prise position apparaît comme un jugement positif sur l'usage qu'un autre locuteurfait du mot : « eh bien je trouve que Fillon a eu raison» (1.3), puis, en fin d'extrait, «François Fillon a raison d'utiliser un mot qui rassure les marchés » (1.10). La justification de cette prise de position, ouverte par le connecteur "parce que» (1.3), repose, comme nous l'avons entrevu, sur le ciblage d'un allocutaire: "tous les grands observateurs" du "monde économique d'aujourd'hui » (1.4), «ceux qui ont de l'argent et ceux qui en prêtent » (1.5-6), « les marchés » (deux occurrences, 1.9-10) et « les financiers internationaux qui prêtent de l'argent y compris à la France » (1.19-20). Or le non-usage du mot « rigueur » est décrit comme négatif dans ses effets ("ne pas être compris», 1.5), tandis que l'usage de ce mot est décrit comme positif : le mot « rassure » l'allocutaire (1.10-12), qui l'« atten[d] » (1.19). On voit se dessiner ici la logique argumentative suivie par Longuet: l'usage du mot est préconisé en raison des effets positifs qu'il exerce sur un type d'allocutaire dont l'opinion est jugée importante au vu de son statut de créancier de la nation. Ce qu'il y a de remarquable dans cet extrait, c'est que la question de l'applicabilité référentielle du mot est également abordée, mais seulement pour être déclarée non décisive sur le plan de l'argumentation: selon Longuet, le fait que le mot soit inapplicable référentiellement ne saurait, dans ce cas particulier, servir d'argument justifiant la conclusion attendue (à savoir qu'il ne faut pas l'utiliser) : « il y a des mots qu'il faut savoir utiliser non pas pour l- pour la réalité française mais parce que vous savez quand on a euh le huit pour cent euh six pour cents de déficit en 2010 c'est pas de la rigueur» (1.7-9, je souligne). Longuet affirme ici clairement que le référent (la « réalité française ») ne peut pas être inscrit dans la classe des référents dénotée par le 
mot, ce qui pourrait apparaitre comme un argument anti-orienté par rapport à la thèse défendue. Dans la perspective du locuteur, cela n'importe guère car l'argument de l'applicabilité référentielle est très largement compensé par l'argument des effets du mot sur l'allocutaire.

\subsubsection{La combinaison possible des deux enchaînements argumentatifs prototypiques}

Nous avons jusqu'à présent envisagé séparément les deux régimes de justification de l'usage ou du non-usage d'un mot, mais il est clair qu'ils peuvent être mobilisés conjointement par un locuteur. Pour illustrer ce cas de figure, nous nous arrêterons sur un dernier extrait (20), tiré d'une interview radiophonique de François Baroin, à l'époque ministre du Budget, par le journaliste Jean-Jacques Bourdin :

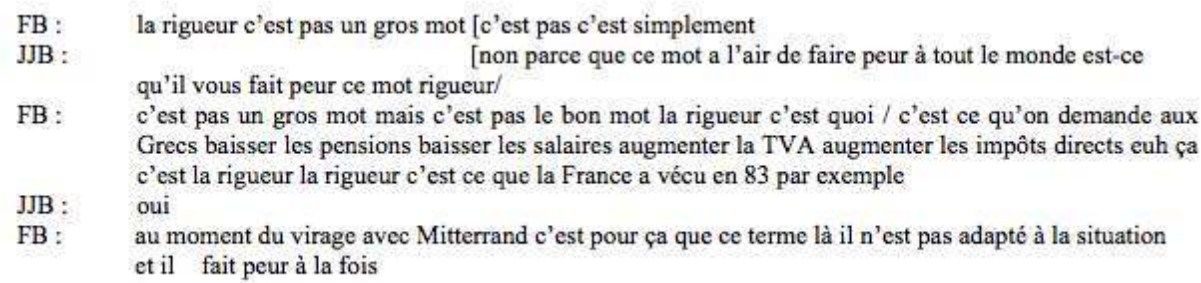

Voyons comment Baroin justifie sa prise de position négative à l'égard du mot, selon laquelle «c'est pas le bon mot» (1.4). La formulation même de cette prise de position ne préjuge en effet pas du type d'argument que le locuteur va solliciter pour la soutenir : qu'un mot ne soit "pas le bon » peut tout aussi bien tenir à des problèmes d'applicabilité référentielle qu'aux contextes qu'il est dialogiquement susceptible d'évoquer pour tel ou tel allocutaire. Baroin propose d'abord une spécification du sens du mot au moyen d'un énoncé définitoire copulatif (« la rigueur c'est quoi / [...] baisser les pensions baisser les salaires augmenter la TVA augmenter les impôts directs ", 1.4-6), assorti de deux exemples de référents auxquels le mot est applicable : «c'est ce qu'on demande aux Grecs » (1.4-5) et « la rigueur c'est ce que la France a vécu en 83 par exemple [...] au moment du virage avec Mitterrand » (1.6-8). Les étapes intermédiaires de l'enchaînement argumentatif qui conduit de la spécification du sens du mot à l'affirmation de son inapplicabilité ("c'est pas le bon mot ", «ce terme là il n'est pas adapté à la situation », 1.8-9) restent ici implicites : n'apparaît ainsi pas, dans l'extrait, l'étape de comparaison du sens spécifié avec les caractéristiques du référent. Quoi qu'il en soit, c'est bien la justification du non-usage du mot par l'argument de l'inapplicabilité référentielle que l'on retrouve ici. Mais Baroin n'en reste pas là : le deuxième exemple qu'il donne d'un référent auquel le mot s'applique («c'est ce que la France a vécu en 83 par exemple [...] au moment du virage avec Mitterrand») lui permet d'activer, de manière dialogique, un contexte antérieur d'emploi supposé prégnant dans la mémoire de l'allocutaire. La référence concerne le changement radical de politique économique opéré à partir du mois de mars 1983 par le gouvernement du Premier Ministre Pierre Mauroy face au déficit budgétaire, à la fuite des capitaux et à la dévaluation du franc. Or ce contexte évoqué par l'usage du mot « rigueur » est décrit comme exerçant des effets négatifs : il « fait peur» (1.9). On voit donc ici que l'argument de la charge dialogique s'ajoute à celui de l'inapplicabilité référentielle pour disqualifier le mot. On ajoutera encore, pour terminer, que l'exploitation du dialogisme par Baroin diffère de celle que l'on a observée dans 
l'argumentation de Longuet (supra, 3.3.2.) : si les deux locuteurs mobilisent le même schème argumentatif, ils n'évaluent pas les effets de l'usage du mot à partir du même type d'allocutaire. Là où Longuet met en avant les acteurs du monde économiqueque l'usage du mot "rassure ", car il leur évoque des contextes d'emploi favorables à leurs intérêts, Baroin semble se concentrer sur les citoyens auxquels l'usage du mot «fait peur ", car il leur évoque des contextes d'emploi défavorables à leurs intérêts.

\section{Conclusion}

Le but de cet article était de poser quelques jalons pour l'étude des querelles de mots : il s'agit, on l'a vu, d'affrontements médiatiques lors desquels un mot est érigé pour une durée limitée en objet du désaccord, ce qui amène les acteurs politiques à formuler une prise de position pour ou contre l'usage de ce mot et à justifier celle-ci au moyen d'arguments. Ces querelles de mots constituent un terrain extrêmement fertile pour une approche qui entend joindre la description des formes du métalangage ordinaire à une analyse du fonctionnement de l'argumentation en contexte politico-médiatique. J'ai travaillé à l'élaboration d'un modèle d'analyse qui tente d'associer une relative économie sur le plan théorique - en ce qu'il met délibérément l'accent sur un nombre limité de processus discursifs et argumentatifs - à un fort potentiel de rentabilité sur le plan empirique. L'examen du corpus de discours rassemblés à partir de la polémique sur le mot « rigueur » offre une première exemplification des trois processus qui, dans l'état actuel de la réflexion, me semblent constitutifs des querelles de mots : le cadrage médiatique de la querelle par les questions et commentaires des journalistes, la prise de position des acteurs politiques quant à l'usage du mot et, enfin, le travail d'étayage argumentatif. Pour que le modèle ici esquissé puisse être affiné et complété, il conviendrait bien sûr de s'intéresser à d'autres querelles de mots similaires ${ }^{21}$ : la résurgence de la polémique sur le même mot en 2011 et 2012 pourrait à l'évidence être envisagée, tout comme des affrontements sur d'autres mots (on pense par exemple à celui qui s'est cristallisé autour du mot « civilisation » suite à la déclaration du ministre de l'Intérieur Claude Guéant le 4 février 2012 selon laquelle « Toutes les civilisation ne se valent pas »).

\section{Conventions de transcription}

- mot interrompu brutalement par un locuteur, troncation de mots

/ intonation montante

$\backslash$ intonation descendante

[ interruption et chevauchement; le crochet précède chacun des segments de discours superposés

\section{BIBLIOGRAPHIE}

Angenot, Marc. 2008. Dialogues de sourds. Traité de rhétorique antilogique (Paris : Mille et Une Nuits) 
Authier-Revuz, Jacqueline. 1995. Ces mots qui ne vont pas de soi. Boucles réflexives et non-coïncidences du dire (Paris : Larousse)

Authier-Revuz, Jacqueline. 2003. « Le fait autonymique : langage, langue et discours. Quelques repères », Authier-Revuz, Jacqueline, Marianne Doury \& Sandrine Reboul-Touré (éds). Parler des mots. Le fait autonymique en discours (Paris : Presses de la Sorbonne Nouvelle), 67-99

Authier-Revuz, Jacqueline. 2004. «La représentation du discours autre : un champ multiplement hétérogène ", Lopez Munoz, Juan, Sophie Marnette \& Laurence Rosier (éds). Le discours rapporté dans tous ses états (Paris : l'Harmattan), 35-53

Authier-Revuz, Jacqueline, Marianne Doury \& Sandrine Reboul-Touré (éds). 2003. Parler des mots. Le fait autonymique en discours (Paris, Presses de la Sorbonne Nouvelle)

Bakhtine, Mikhail. 1978. Esthétique et théorie du roman (Paris : Gallimard)

Barbet, Denis. 2009. Grenelle : histoire politique d'un mot (Rennes : PUR)

Bres, Jacques. 2005. « Savoir de quoi on parle : dialogue, dialogal, dialogique ; dialogisme, polyphonie... », Bres, Jacques et al. (éds). Dialogisme et polyphonie. Approches linguistiques (Bruxelles : De Boeck), 47-63

Bres, Jacques \& Aleksandra Nowakowska. 2006. «Dialogisme : du principe à la matérialité discursive », Perrin, Laurent (éd.), Le sens et ses voix (Metz : Recherches linguistiques), 21-48 Cislaru, Georgeta etal. (éds). 2007. L'acte de nommer. Une dynamique entre langue et discours (Paris : Presses de la Sorbonne Nouvelle)

Détrie, Catherine et al. (éd.). 2001. Termes et concepts pour l'analyse du discours. Une approche praxématique (Paris : Champion)

Dufour, Françoise. 2011. « Développement durable, humain, La cohérence discursive des contradictions », Mots 96, 81-97

Dufour, Françoise et al. (éds). 2005. La nomination : quelles problématiques, quelles orientations, quelles applications? (Montpellier, Université Paul Valéry-Montpellier 3)

Hekmat, Ida. 2011. « La polémicité des formules “ choc des civilisations ”et “ Kampf der Kulturen " : une étude contrastive ", Burger, Marcel et al. (éds), La parole politique en confrontation dans les médias (Bruxelles : De Boeck), 89-107

Julia, Catherine. 2001. Fixer le sens. La sémantique spontanée des gloses de spécification du sens (Paris : Presses de la Sorbonne Nouvelle)

Kerbrat-Orecchioni, Catherine. 2005. Le discours en interaction (Paris: Colin)

Krieg-Planque, Alice. 2003. «Purification ethnique ». Une formule et son histoire (Paris : CNRS éditions)

Krieg-Planque, Alice. 2009. La notion de formule en analyse du discours : cadre théorique et méthodologique (Besançon : Presses Universitaires de Franche-Comté)

Krieg-Planque, Alice. 2010. « La formule "développement durable" : un opérateur de neutralisation de la conflictualité », Langage et Société 134, 5-29

Krieg-Planque, Alice. 2011. «Les “ petites phrases ” : un objet pour l'analyse des discours politiques et médiatiques », Communications et langages 168, 23-41

Longhi, Julien. 2008. Objets discursifs et doxa. Essai de sémantique discursive (Paris : l'Harmattan)

Martin, Robert. 1983. Pour une logique du sens (Paris : PUF) 
Martin, Robert. 1990. « La définition "naturelle" ", Chaurand, Jacques \& Francine Mazière (éds), La définition (Paris : Larousse), 86-95

Micheli, Raphaël. 2010. « Argumentation et réflexivité langagière : propositions pour l'étude de l'usage argumentatif des définitions », Verbum XXXII : 1, « L'inscription langagière de l'argumentation », 143-161

Micheli, Raphaël. 2011. «Quand l'affrontement porte sur les mots en tant que mots : polémique et réflexivité langagière », Semen31, « Polémiques journalistiques et médiatiques », 97-112

Née, Emilie. 2009. Sûreté, sécurité, insécurité. D'une description lexicologique à une étude du discours de presse : la campagne électorale 2001-2002 dans Le Monde, Thèse de Doctorat, Université Paris-3, [en ligne], http://syled.univ-paris3.fr/individus/emilie-nee/3.html

Ollivier-Yanniv, Caroline. 2011. «Les "petites phrases" et “éléments de langage” : des catégories en tension ou l'impossible contrôle de la parole par les spécialistes de la communication ", Communications et langages 168, 57-68

Plantin, Christian. 1996. L'argumentation (Paris : Seuil, coll. « Mémo »)

Plantin, Christian. 2002. « Analyse et critique du discours argumentatif », Amossy, Ruth \& Roselyne Koren (éds), Après Perelman(Paris : L'Harmattan), 229-263

Rey-Debove, Josette. 1978. Le métalangage. Etude linguistique du discours sur le langage (Paris : Le Robert)

Riegel, Martin. 1987. « Définition directe et indirecte dans le langage ordinaire : les énoncés définitoires copulatifs », Langue Française 73, 9-53

Riegel, Martin. 1990. «La définition, acte du langage ordinaire. De la forme aux interprétations ", Chaurand, Jacques \& Francine Mazière (éds), La définition (Paris : Larousse), 97-110

Schiappa, Edward. 1993. « Arguing about Definitions », Argumentation 7 : 4, 403-417

Schiappa, Edward. 2003. Defining Reality. Definitions and the Politics of Meaning (Carbondale: Southern Illinois University Press)

Véniard, Marie. 2007. La nomination d'un événement dans la presse quotidienne nationale. Une étude sémantique et discursive : la guerre en Afghanistan et le conflit des intermittents dans Le Monde et Le Figaro, Thèse de doctorat, Université Paris 3, [en ligne], http://syled.univ-paris3.fr/individus/ marie-veniard/3.html

Walton, Douglas. 2006. Fundamentals of Critical Argumentation (Cambridge : CUP)

Walton, Douglas \& Fabrizio Macagno. 2008. «The Argumentative Structure of Persuasive Definitions ", Ethical Theory and Moral Practice 11, 525-549

Walton, Douglas \& Fabrizio Macagno. 2009. « Reasoning from Classifications and Definitions », Argumentation 23: 1, 81-107

Zarefsky, David. 2006. «Strategic Maneuvering through Persuasive Definitions : Implications for Dialectic and Rhetoric », Argumentation $20: 4,399-416$

\section{NOTES}

1. C'était le cas lors de la polémique qui a opposé en mai 2008 Bertrand Delanoë et Ségolène Royal-alors en concurrence pour la succession de François Hollande à la tête du Parti socialiste - autour des mots « libéral »/« libéralisme »: Delanoë incitait alors ses camarades à se 
réapproprier ces mots, ce qui lui valut des critiques acerbes de la part de Royal (voir Micheli 2011 ; Longhi 2008 pour une analyse sémantique des mêmes mots dans un corpus politique plus ancien).

2. Au vu de l'abondance et de la diversité des travaux, cet état de la recherche ne peut qu'être esquissé ici.

3. On pense ici principalement au travail fondateur de Rey-Debove (1978) sur le métalangage en tant que "sous-système cohérent » et à l'œuvre considérable d'Authier-Revuz qui cartographie les différentes zones de la réflexivité langagière : auto-représentation du discours en train de se faire (1995) et représentation du discours autre notamment (2004).

4. Dans le cadre d'un intérêt pour le processus de nomination(Cislaru et al. 2007 ; Dufour et al. 2005), plusieurs études récentes suivent un mot (ou une séquence lexicale figée de type "formule ») à travers un corpus discursif donné (en général médiatique) pour en décrire le comportement linguistique et en éclairer les enjeux socio-politiques : on a par exemple exploré des mots comme « sûreté »/ « sécurité »/ « insécurité » (Née 2009), « crise »/ « guerre »/ « conflit » (Véniard 2007), « intermittent » (Longhi 2008; Véniard 2007), «Grenelle» (Barbet 2009) ou des formules comme "purification ethnique " (Krieg-Planque 2003), « développement durable» (Dufour 2011; Krieg-Planque 2010) ou encore « choc des civilisations» (Hekmat 2011). De telles études sont par nature attentives à l'activité métalinguistique que les locuteurs déploient à propos du mot (ou de la formule) qui fait l'objet de l'analyse.

5. A ce titre, on peut même souligner, avec Plantin, que les approches normatives semblent parfois habitées par la tentation inavouée de «sort[ir] du langage » ordinaire afin d'en arriver à la « bonne » argumentation, univoque et rationnelle (2002: 235-236).

6. Si l'on excepte les travaux récents sur la définition en tant que schème argumentatif (Micheli 2010 ; Schiappa 2003 ; Walton et Macagno 2008 et 2009 ; Zarefsky 2006), ainsi que des remarques générales sur les "verbal disputes» (Walton 2006), sur les « débats » qui " achoppent sur les mots des autres [et sur] les définitions avancées » (Angenot $2008: 158$ ) ou encore sur le fait que, dans certains débats, la « discussion sur la nature de l'objet n'est pas séparable de la discussion sur son nom» (Plantin 1996: 63). On mentionnera encore - même si elle ne porte pas directement sur l'argumentation - la réflexion de Kerbrat-Orecchioni sur les «négociations conversationnelles », lorsque celles-ci tournent en "désaccords sur l'emploi des mots», en « négociations sur les signes » (2005: 131).

7. Voir les travaux récents en communication politique sur des catégories comme les «petites phrases » (Krieg-Planque 2011) et les « éléments de langage » (Ollivier-Yaniv 2011).

8. Fichier audio accessible à http://www.rtl.fr/actualites/economie/article/michel-sapin-surrtl-c-est-une-rigueur-gigantesque-qui-est-programmee-5940149834

9. Fichier audio accessible à http://www.europe1.fr/MediaCenter/Emissions/Le-buzz-politique/ Sons/Cope-la-rigueur-ne-lui-fait-pas-peur-190937/

10. Vidéo accessible à http://www.auboisementcorrect.com/4690-Francois-Baroin-La-rigueurce-n.html

11. Vidéo accessible à http://www.dailymotion.com/video/xe0upj_interview-de-nicolassarkozy-sur-fr_news

12. Vidéo accessible à http://www.europe1.fr/Politique/Des-souvenirs-mais-pas-desous-236074/

13. La vidéo était accessible à http://www.lemouvementpopulaire.fr/actualites/Il-ne-faut-pasconfondre-plan-de-rigueur-et-gouvernement-rigoureux-5746.html

14. http://www.slate.fr/story/21495/jean-francois-cope-cachez-cette-rigueur-que-je-nesaurais-voir

15. Sur cette gestion sarkozienne de la famille dérivationnelle " rigueur »/ « rigoureux », lire la très intéressante chronique que lui a consacrée la linguiste Emilie Née (http:// 
blogs.mediapart.fr/blog/emilie-nee/030810/l-homme-honnete-l-homme-juste-l-hommerigoureux)

16. Il va sans dire que dans les réalisations textuelles effectives, l'ordre d'apparition de ces étapes peut varier.

17. De tels énoncés sont susceptibles de prendre des formes très diverses - point que l'on ne peut développer ici. Les travaux de Martin (1983 et 1990) et de Riegel (1987 et 1990) proposent une typologie qui distingue entre les énoncés définitoires ouvertement métalinguistiques (de type désignatif - « le mot $X$ désigne/est le nom de $Y$ » - ou interprétatif - « Le mot X signifie/veut dire $Y »)$ et les énoncés définitoires copulatifs établissant une relation d'équivalence référentielle au moyen du verbe «être» («Un/le X (c')est un/le Y»). Voir aussi l'étude de Julia (2001) sur les gloses de spécification du sens (« au sens propre », " dans tous les sens du mot », etc.)

18. Plantin identifie un schème argumentatif qu'il nomme "argumentation par la définition » (1996 : 52), qui ressemble fort à celui que nous représentons ici - si ce n'est que la conclusion ne porte pas sur l'usage du mot. Voir aussi Schiappa (2003: 109) sur l'argumentation à partir d'une définition (" argument from definition » - par opposition à l'« argument about a definition »).

19. Je n'entre donc pas ici dans toute la complexité du phénomène, particulièrement en ce qui concerne le problème de distinguer les différents types de dialogisme - interdiscursif et interlocutif - et de les corréler à des marques langagières précises (voir notamment, parmi l'abondante littérature sur la question, Bres 2005 ; Bres et Nowakowska 2006).

20. L'enchaînement argumentatif que j'envisage ici a pour caractéristique d'exploiter conjointement le raisonnement pratique (à travers l'argumentation par les conséquences qu'entraîne l'usage du mot sur un allocutaire) et la question du dialogisme (à travers les contextes antérieurs d'emploi que l'usage du mot est susceptible d'évoquer chez l'allocutaire). Ces deux dimensions peuvent ne pas aller de pair dans certains cas: on peut par exemple tout à fait imaginer un enchaînement argumentatif qui tire uniquement parti des effets (positifs ou négatifs) de l'usage du mot sur l'allocutaire, sans que ces effets soient rapportés au dialogisme.

21. L'intérêt d'une telle approche comparative serait par exemple de voir dans quelle mesure les diverses composantes de l'argumentation pour ou contre l'usage d'un mot que l'on a recensées ici sont susceptibles d'être pondérées différemment dans chacune des querelles considérées. On pourrait ainsi imaginer que les participants à une querelle de mots donnée mettent un accent tout particulier sur la spécification du sens du mot, et s'intéressent moins à son applicabilité référentielle et à son dialogisme. A l'inverse, d'autres querelles seraient susceptibles de se concentrer davantage sur le dialogisme du mot que sur son (ou ses) sens, etc.

\section{RÉSUMÉS}

L'objectif de cet article est de contribuer à la description d'un type particulier d'affrontement qui met régulièrement aux prises les acteurs politiques dans l'espace médiatique contemporain : la querelle de mots. Dans ce genre de cas, un mot particulier est érigé en objet du désaccord, sous l'effet d'un travail de cadrage médiatique opéré par les journalistes : les acteurs politiques sont amenés à prendre position quant à son usage et à justifier leur prise de position au moyen d'arguments. On esquisse un modèle d'analyse de ces querelles de mots et l'on teste ce modèle sur un corpus de discours constitué à partir d'une polémique ayant opposé plusieurs acteurs politiques français autour $\mathrm{du}$ mot «rigueur» entre mai et juillet 2010. Sur le plan méthodologique, la démarche se situe à la croisée de la linguistique, des théories de 
l'argumentation et des sciences de la communication et des médias : elle articule l'étude des formes du métalangage ordinaire à celle du fonctionnement argumentatif des discours et des enjeux contemporains de la médiatisation du politique.

This paper aims to contribute to the description of a particular type of confrontation, often taking place between political actors in contemporary media, namely verbal disputes. In such confrontations, a particular word becomes the very object of disagreement. Under the effect of a framing process led by journalists, political actors are invited to take a stand as to whether the word should or should not be used and to justify their position by means of arguments. I propose a model of analysis for verbal disputes, and I test it using a data set composed of a controversy involving several French political actors over the use of the word "rigueur" between May and July, 2010. Methodologically, this paper is influenced by linguistics, argumentation theory and media studies. The paper is focused on describing forms of ordinary metalanguage as well as argument schemes, and in reflecting on the mediatisation of politics.

\section{INDEX}

Mots-clés : argumentation, discours politique, médias, métalangage

Keywords : argumentation, media, metalanguage, political discourse

\section{AUTEUR}

\section{RAPHAËL MICHELI}

Université de Lausanne 